cell checkpoints and aberrant plasmablast development in SLE has not been previously characterised. Iberdomide (a cereblon modulator) known to induce the degradation of transcription factors IKZF1 and IKZF3 is being explored as a therapeutic target for SLE. The aim of this study was to utilise iberdomide to evaluate the effect of inhibition of IKZF1 and IKZF3 on transcriptional programmes underlying B cell differentiation, gene expression and immunoglobulin production in SLE B cells.

Methods CD19 +B cells were isolated from peripheral blood of 25 SLE patients and stimulated with IL-2, IL-10, IL-15, CD40L and TLR7 ligand Resiquimod for 5 days to induce plasmablast differentiation. In separate studies, B cells were treated from the outset with iberdomide $(10 \mathrm{nM})$ or vehicle and subsequently differentiated, or differentiated plasmablasts (day 4) were treated with iberdomide or vehicle for 18 hour. Treated plasmablasts underwent fluorescence-activated cell sorting (FACS), and $\operatorname{IgG} / \operatorname{IgM}$ secretion analysed with ELISA. FACS-sorted CD27-IgD+naïve $\mathrm{B}$ cells and CD20lowCD27 $+\mathrm{CD} 38+$ plasmablasts were subjected to bulk ultra-low input RNA-seq along with matched baseline B cells. Unsupervised clustering, differential gene expression and pathway analysis were performed on transcriptome data.

Results Day 0 iberdomide $(n=9)$, but not day 4 iberdomide $(n=16)$, significantly reduced the CD20lowCD27+CD38+plassmablast numbers following cell culture $(p=0.03)$. Similarly, Day 0 iberdomide significantly decreased supernatant $\mathrm{IgG} / \mathrm{IgM}$ concentrations $(p=0.050$ and 0.017 , respectively), but not day 4 iberdomide. RNA-seq of sorted naïve B cells and plasmablasts cultured with day 4 iberdomide demonstrated significant differential gene expression in both populations (400 and 461 differentially modulated genes in naïve $\mathrm{B}$ cells and plasmablasts, FDR-adjusted $\mathrm{p}<0.05)$. Pathway analysis showed that IKZF1/IKZF3 inhibition resulted in downregulation of JAKSTAT signalling downstream of IL12 $(\mathrm{FDR}=7.92 \mathrm{E}-04)$, IL12 signalling $(\mathrm{FDR}=0.0014)$, and $\mathrm{p} 53$ signalling regulation of cell death $(\mathrm{FDR}=0.0043)$ and showed a trend to upregulation of RUNX1 signalling and Rho GTPase cycle.

Conclusions Iberdomide exposure significantly blocked SLE B cell differentiation into plasmablasts, but did not alter fully differentiated plasmablast viability, confirming the role of IKZF1 and IKZF3 in the process of B cell differentiation into aberrant plasmablasts in SLE. Our study demonstrates that IKZF1 and IKZF3 inhibition results in differential expression of key B cell development transcriptional gene modules in both SLE naïve B cells and plasmablasts.

Funding Source(s): This study was supported by research funding from Celgene Corporation.

\section{UNDERSTANDING SYSTEMIC LUPUS ERYTHEMATOSUS. A QUALITATIVE STUDY OF WOMEN WITH INACTIVE DISEASE}

Andreia Carniell, Simone Appenzeller*, Lilian Costallat, Egberto Turato. University of Campinas

\subsection{6/lupus-2019-Ism.236}

Background Systemic lupus erythematosus (SLE) is a chronic and cyclic disease with an autoimmune characteristic caused by a combination of environmental, hereditary, stress or congenital predispositions. The acute period of the disease is experienced by the patients as debilitating and of adaptive psychological changes. In the period of remission of the clinical symptoms of the disease, other adaptive psychological components are used as a way of dealing with the limitations imposed by the disease. Objective: To verify how people with systemic lupus erythematosus use adaptive psychological resources to deal with the disease in this asymptomatic period.

Methods This qualitative study was used to study 9 selected women after the application of SLEDAI (Systemic Lupus Erythematosus Systemic Activity Index) in the post-consultation period with scores equal to or less than 3. Subsequently, the first author's psychologist The qualitative research studies proposed the understanding of the phenomena observed in the scientific environment. The data collected through the recording of participants' speeches were analyzed through content analysis, using the theoretical framework of health psychology. The intention is always to understand the manifestation of something in a group, without the causal intention, only then to give visibility to new strategies both for therapeutic interventions and for new generalizable scientific studies. University Hospital of Southeastern Brazil. In this qualitative study, the strategy of saturation was used to calculate sample size and the number of individuals was held when we verified similarity in responses.

Results 9 female participants were selected. In the speeches of participants, the following topics were identified: Do not think about the existence of the disease; The return to daily activities; Living with other people; The perception of the current body.

Conclusions The perception of the possibility of reactivation of the disease, allows these participants a constant vigil of occasional symptoms that arise. It is such a way to have emotional control over the occasional symptoms that arise. Reviving in anticipation all the trajectory of a possible aggravation of the disease. It is the fear and rationalization of the possibility of reactivation of Systemic Lupus Erythematosus, which allows these participants to have adherence to the continuous treatment in this period.

Funding Source(s): None

\section{CELL BOUND COMPLEMENT ACTIVATION PRODUCTS IN COMBINATION WITH LOW COMPLEMENT C3 OR C4 HAVE HIGH DIAGNOSTIC YIELD IN SYSTEMIC LUPUS ERYTHEMATOSUS}

${ }^{1}$ Thierry Dervieux*, ${ }^{2}$ Daniel J Wallace, ${ }^{3}$ Chaim Putterman, ${ }^{4}$ Cristina Arriens, ${ }^{5}$ Kenneth C Kalunian, ${ }^{6}$ Elena M Massarotti, ${ }^{1}$ Roberta Vezza Alexander, ${ }^{1}$ Claudia Ibarra, ${ }^{7}$ Rosalind Ramsey-Goldman, ${ }^{1}$ Arthur Weinstein, ${ }^{8}$ Sonali Narain, ${ }^{9}$ Amit Saxena, ${ }^{10}$ Christopher E Collins, ${ }^{11}$ Joseph M Ahearn, ${ }^{11}$ Susan Manzi. ${ }^{1}$ Exagen; ${ }^{2}$ Cedars-Sinai Medical Center, University of California, Los Angeles, CA, USA; ${ }^{3}$ Albert Einstein College of Medicine and Montefiore Medical Center; ${ }^{4}$ Oklahoma Medical Research Foundation; ${ }^{5}$ University of California at San Diego School of Medicine; 'Brigham and Women's Hospital; ${ }^{7}$ Northwestern University Feinberg School of Medicine; ${ }^{8}$ Hofstra Northwell School of Medicine; ${ }^{9}$ New York University School of Medicine; ${ }^{10}$ MedStar Washington Hospital Center; ${ }^{11}$ Lupus Center of Excellence Allegheny Health Network

\subsection{6/lupus-2019-Ism.237}

Background Cell Bound Complement Activation Products (CBCAPs), are stable form of classical complement activation, exvivo, and sensitive and specific marker of SLE. In the present study, we sought to compare the performances of CB-CAPs to gold standard low complement C3 or C4. 


\begin{tabular}{|c|c|c|c|c|c|c|}
\hline & Sensitivity & Specificity & Youden & $\begin{array}{c}\text { AUC } \\
(\mathrm{CI} 95 \%)\end{array}$ & $\begin{array}{c}\text { OR } \\
(\mathrm{CI} 95 \%)\end{array}$ & AIC \\
\hline Low C3 $(<81 \mathrm{mg} / \mathrm{dl})$ & $27 \%$ & $97 \%$ & 0.240 & $\begin{array}{c}0.620 \\
(0.599,0.641)\end{array}$ & $\begin{array}{c}10.9 \\
(6.28,18.91)\end{array}$ & 1200 \\
\hline Low C4 (<12.9 mg/dl) & $27 \%$ & $97 \%$ & 0.235 & $\begin{array}{c}0.618 \\
(0.596,0.639)\end{array}$ & $\begin{array}{c}9.57 \\
(5.67,16.15)\end{array}$ & 1206 \\
\hline Low C3 or low C4 & $38 \%$ & $93 \%$ & 0.313 & $\begin{array}{c}0.656 \\
(0.632,0.681)\end{array}$ & $\begin{array}{c}8.34 \\
(5.55,12.53)\end{array}$ & 1174 \\
\hline Abnormal EC4d (>14 net MFI) & $43 \%$ & $92 \%$ & 0.350 & $\begin{array}{c}0.675 \\
(0.650,0.700)\end{array}$ & $\begin{array}{c}8.67 \\
(5.9,12.72)\end{array}$ & 1153 \\
\hline Abnormal BC4d ( $>60$ net MFI) & $50 \%$ & $94 \%$ & 0.436 & $\begin{array}{c}0.718 \\
(0.693,0.743)\end{array}$ & $\begin{array}{c}14.95 \\
(9.81,22.77)\end{array}$ & 1082 \\
\hline Abnormal EC4d or BC4d & $62 \%$ & $88 \%$ & 0.492 & $\begin{array}{c}0.746 \\
(0.719,0.772)\end{array}$ & $\begin{array}{c}11.05 \\
(7.95,15.37)\end{array}$ & 1067 \\
\hline
\end{tabular}

Methods All subjects $(\mathrm{n}=1200)$ were adults $(18$ years $)$ and enrolled from multiple academic Centers in the United States. All SLE fulfilled the 1997 ACR criteria for SLE $(n=498)$. Patients with Other Rheumatic Diseases $(n=450)$ consisted of 189 rheumatoid arthritis, 88 Sjogrens, 90 Fibromyalgia and 83 patients with other connective tissues diseases. A group of healthy normal individuals was also enrolled $(n=252)$. Abnormal CB-CAPs status (EC4d or BC4d $>99$ th percentile of normal) were determined using flow-cytometry. Complement C3 and C4 levels were determined using immunoturbidimetry (Binding Site, San Diego, CA) assay kits. Performances of the markers, either alone or in combination to distinguish SLE from other rheumatic diseases and controls were established using Sensitivity, Specificity, Odds Ratio and Area under the Curve (AUC) of the Receiver Operating Characteristic curve (ROC). Youden Index (Sensitivity + Specificity - 100) was also calculated. The combination of 4 complement marker abnormalities were also evaluated using logistic regression and unweighted composite score cumulating the presence of these abnormalities was calculated.

Results Abnormal CB-CAPs status yielded 62\% sensitivity with $88 \%$ specificity in distinguishing SLE from the group of patients with other diseases (table 1). Youden Index was 0.492 \pm 0.027 . Low C3/C4 status yielded $38 \%$ sensitivity and $93 \%$ specificity in distinguishing SLE from the group of patients with other diseases. Youden Index was $0.313 \pm 0.025$ for Low $\mathrm{C} 3$ or $\mathrm{C} 4$ and significantly lower than Youden score associated with abnormal CB-CAPs $(p<0.01)$. Specificity of Low C3/C4 and abnormal CB-CAPs in distinguishing SLE from normal healthy individuals was $93 \%$ and $99 \%$, respectively. AUC was also significantly higher with $\mathrm{BC} 4 \mathrm{~d}(0.718)$ than with low $\mathrm{C} 3$ (0.620; p <0.01), low C4 $(0.618 ; \mathrm{p}<0.01)$ and Low C3 or C4 status $(0.656 ; \mathrm{p}<0.01)$. A composite score (unweighted) cumulating all 4 abnormalities, was higher in SLE $(1.47 \pm 0.06)$ than disease control group $(0.21 \pm 0.02)(\mathrm{p}<0.01)$ and normal healthy individuals $(0.01 \pm 0.02) \quad(\mathrm{p}<0.01)$. The complement scoring system yielded higher AUC (0.812), higher OR (36.0 CI95\%: 18.8-69.0), lower AIC (1037) and greater R2 (0.403) than any other combinations
Conclusions Our data suggests that CB-CAPs have greater diagnostic yield than low complement $\mathrm{C} 3 / \mathrm{C} 4$. The combination of these complement abnormalities in composite complement score has high yield in distinguishing SLE from other rheumatic diseases and normal healthy individuals.

Funding Source(s): Exagen.

\section{LEUKOCYTE IMMUNOGLOBULIN-LIKE RECEPTOR A3 (LILRA3) PROMOTES LUPUS-LIKE DISEASE IN A MURINE LUPUS MODEL}

Jianping Guo*, Yuxuan Wang, Yundi Tang. Peking University, People's Hospital

\subsection{6/lupus-2019-Ism.238}

Background Leukocyte immunoglobulin-like receptor A3 (LILRA3) is a secreted protein belongs to LILR family. Our research group previously reported that the functional LILRA3 is a novel genetic risk for multiple autoimmune diseases including systemic lupus erythematosus (SLE). However, the function of LILRA3 in development of lupus is unclear. The bm12 model is a chronic graft-versus-host disease (cGVHD) model characterized by the lupus-like syndrome with cell subtype alteration and autoantibody production. To functionally study the role of LILRA3 in lupus pathogenesis, we constructed the LILRA3 knock-in mice and assessed the clinical manifestation and immune responses in bm12 model.

Methods Human LILRA3 gene (Gene ID: 11026) was inserted into Rosa26 allele in C57BL/6 (B6) mice based on Cas9/ sgRNA system. The cGVHD was induced by an intraperitoneal injection of the bm12 donor splenocytes into recipients, either B6 wild-type or knock-in mice. Mice were sacrificed on day 14 and 28. Flow cytometry was used to analyze frequencies of immunocytes from mice splenocytes. Enzyme-linked immunosorbent assay was applied to detect antibodies in serum.

Results Compared with the wild-type mice, the LILRA3 knock-in mice displayed a more severe immune response on 